\title{
ON PROTOTYPING METHODS TO LEVERAGE NON-RIGID MATERIALS IN THE EARLY STAGES OF ENGINEERING DESIGN
}

\author{
M. Auflem ${ }^{1, \otimes}$, H. H. Boe ${ }^{2}$, J. F. Erichsen ${ }^{1}$ and M. Steinert ${ }^{1}$ \\ ${ }^{1}$ Norwegian University of Science and Technology, Norway, ${ }^{2}$ CERN Idea Square, Switzerland \\ $\triangle$ mariuauf@stud.ntnu.no
}

\begin{abstract}
Prototyping has been shown important to facilitate learning, inform decisions and to communicate ideas in engineering design. However, it is not evident which methods, tools and materials to use, as prototyping is practised differently across development contexts, and stages. In the early stages of design, different choices in prototyping methods, tools and materials all affect prototyping outcome. This paper is focused on prototyping methods in the context of early stages of design and attempts to highlight identified strengths and limitations of using non-rigid materials for prototyping.
\end{abstract}

Keywords: prototyping, design tools, early design phase, case study

\section{Introduction}

In engineering design research, prototyping has been shown important to facilitate learning, inform decisions and to communicate ideas (Lauff et al., 2018). However, it is not evident which methods, tools and materials to use, as prototyping is practised differently across contexts, disciplines and stages of development. The design engineer has to prioritise various trade-offs in order to effectively create focused prototypes with purpose and intention (Bryan-Kinns and Hamilton, 2002; Houde and Hill, 1997; Lim et al., 2008; Menold, 2017).

Although there are many definitions of prototypes in engineering design research, as discussed by Jensen et al. (2016). this paper uses the definition from Schrage (1993), who states that prototypes are created to answer questions. Consequently, all objects could serve as prototypes if they convey some attribute of the conceptual idea to the designer (Buchenau and Suri, 2000). Subsequently, prototyping is defined as a learning activity (Leifer and Steinert, 2011), both cognitive and physical, where the goal is to generate tangible insights in form of prototypes (Auflem et al., 2019).

In the early stages of engineering design, different choices in prototyping methods, tools and materials all affect prototyping outcome. This paper is focused on the use of materials in the context of early stages of engineering design and attempts to highlight identified strengths and limitations of prototyping methods using non-rigid materials. Flexible and semi-flexible materials (further referred to as non-rigid materials), have enabled product opportunities within various fields and disciplines. However, the authors argue that in product development, and especially in the early stages of design, there is a lack of research, tools and, examples on how to leverage these non-rigid materials effectively.

In this paper non-rigid materials will for all intents and purposes be considered materials allowing elastic deformations even when subjected to large deflections. While non-rigid materials are not new 
to the field of engineering, availability of materials and manufacturing technologies have made them a relevant topic in research and recent developments. Medical technology, soft robotics, biomimicry and compliant mechanisms are some areas where non-rigid materials have had a substantial impact and introduced new functional opportunities.

In engineering design, complex material properties, deformations and/or geometries could all make design and dimensional tasks challenging. Physical computation, as described by Foehr et al. (2015), is shown to be one way of addressing such complex material characteristics and concepts. Here, nonlinearity as a result of large deformations caused numerical computations to diverge- and crash- by having too many unknown parameters. A rapid prototype could in this case be used to reduce the complexity of such computational task by solving, or informing, one or several of the unknown parameters by physical experimentation. Kriesi et al. (2016) presents a similar approach in exploring the complex nature of injection molding processes by prototyping. By rapidly creating molds for a simple desktop injection moulding machine, they could iteratively test and learn about functional requirements for mould-designs, without the need of tedious and complex simulations. Other examples involve physical modelling of manufacturing techniques using plasticine as a proxy material for metal. Common for these cases is how prototyping complex materials and/or conceptual models could enable fast learning without tedious simulations or numerical models.

As prototypes are created and evaluated trough testing, working in iterative cycles could enable concepts to adapt as insights get revealed to the designers. This could reduce the risk of costly rework later in the development. In this process, the creation of prototypes is a crucial activity that facilitates generation of new ideas (ideation), divergence, as well as making these ideas tangible and testable in order to inform further design iterations (Dow et al., 2011). This generative role of prototyping activities is important for designers to diverge, exploring multiple design alternatives and avoid prematurely fixating on concepts or designs (Viswanathan and Linsey, 2011). Hence, rapid and iterative prototyping is considered a crucial tool when exploring uncharted solution spaces in the early stages of engineering design (Camburn et al., 2013).

From these principles, this paper will present similar approaches to physical modelling, and evaluate methods of iterative prototyping to generate fast tangible insights. This will be done to address the challenges of doing concept generation, exploring solution space, and more specifically to leverage non-rigid materials in the early stages of engineering design. In this context the paper will focus on specific design activities, namely prototyping methods to explore and leverage non-rigid materials, as an alternative to evaluating prototyping outcome, purpose and the attributes of prototypes (Jensen et al., 2015). With prototyping being defined as cycles of designing, building and testing of prototypes, the method used to create prototypes is of interest to better understand the activities in design projects.

Further, how the various methods utilized, affect design outcome (prototype attributes), and performance, (build time, facilitating design iterations, and design freedom). By researching such prototyping methods, the authors seek to aid designers in doing strategic decisions when selecting approach to create non-rigid prototypes in the early design stages.

The methods considered in this paper will be illustrated through individual cases, gathered from different development projects. Hence, it is of interest how the different methods affect both prototyping activities and outcome. The presented cases are all projects in early design stages focused towards concept generation and evaluation using prototypes. By this, the paper aims to exemplify various prototyping methods, utilizing tools and technologies to leverage non-rigid materials. These are methods that could help generate fast tangible insights through the designing, building, and testing of physical non-rigid prototypes.

\section{Prototyping methods in early design}

In this section, four prototyping methods for generating non-rigid concepts are illustrated through four project cases. Common for these projects is the development context of early design and potentials for exploring non-rigid concept proposals. As prototypes are created with varying intent and utilized differently across projects, the success criteria for each prototype vary subsequently. Hence, this paper attempts to evaluate the prototyping methods used, rather than the physical artefacts created. By this, 
revealed insights could support designers in effectively deploy methods to generate prototypes with non-rigid abilities. To discuss the presented prototyping methods, the following five parameters are used to describe how the prototyping activity and outcome in terms of prototypes are affected by the method utilized.

- $\quad$ Speed

- Modifications

- Design freedom

- Fidelity

- Resolution

The two initial parameters, speed and modifications, are considering how well a given method enables rapid and iterative working modes in early design. This is important in obtaining fast insights, trough design-build-test cycles when exploring new solution spaces (Leifer and Steinert, 2011). Speed is here used to describe the time per prototype iteration. In the context of tangible insights, time per prototype iteration is of interest because it can help describe if and how a method enables fast learning. Modifications discusses how a method facilitates continuous alterations or if a prototype is hard to change or adapt to revealed insights.

Design freedom evaluates the geometrical, material, and size constraints of any given method. Hence, how workable a method is, and if it can easily adapt to new design ideas, allow for design alterations, or for complex design ideas to be realized. Fidelity discusses the obtainable degree of closeness to eventual design with a given method while resolution discusses the obtainable amount of detail with a given method (Houde and Hill, 1997).

\subsection{Cases}

The following sections describe four different methods of fabricating non-rigid prototypes trough individual case examples. These cases ranges from low-resolution concept generation for flexible animatronic robots, to creating a proof-of-concept prototype to be tested with ultrasound imaging equipment. The methods considered in these cases have only utilized one, or a limited number, of materials and machining/fabrication possibilities. While all the cases concern different materials and fabrication methods, they are not intended as a representative selection for other methods. However, the presented methods are four distinct different alternatives, that visualize a span of tools and technologies that could help leveraging non-rigid materials in prototyping.

\subsection{Soft prototyping}

Soft Prototyping is in engineering design known to ease both creative efforts, team engagement and in avoiding sunk cost effects (Dow et al., 2009). Hence, it is often the initial steps of exploratory prototyping activities in new projects. This method enables the generation of low resolution and low fidelity prototypes, that can be easily modified, tested and discarded based on fast insights. Foam, paper, rubber, and fabric are some commonly available materials that are used in this way of prototyping as they can be easily modified using simple hand-tools. Similarly, ease of use makes glue, tape, needles and ropes important tools to have in the toolbox as it enables rapid assembly and continuous alterations of prototypes.

In the context of non-rigid materials, the project case for soft prototyping is the conceptual design of a soft actuator for animatronic robots. This project had specific demands as it required a cylindrical tube to change its shape in order to simulate a human airway. This meant having control over deformation, not introducing rigid elements, as well as having the opportunity to occlude the cylindrical airway shape as seen in Figure 1.

In this project, physical alterations and iterating different functional principles, was vital in order to visualize and generate solutions. Using simple materials such as flexible foam and plastic film, the designers could realize ideas and test them without spending time on Computer-Aided Design (CAD) or machining. In addition, rapid design changes and iterations enabled large throughput of potential concepts. While prototype resolution and fidelity remained low, the designers could quickly test out principles for both the actuation as well as the geometrical constraints of the simulated airway. 

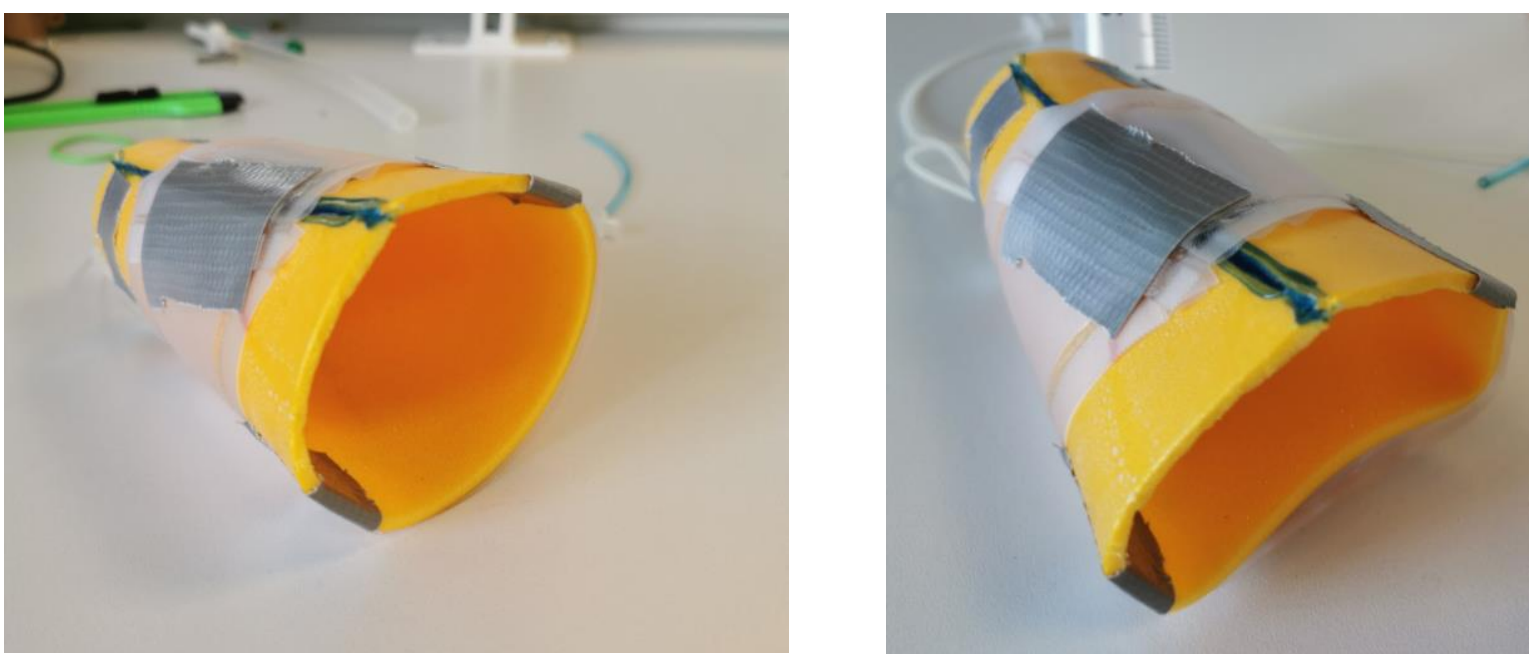

Figure 1. Cylindrical airway shape built using soft prototyping

This case showed the importance of exploratory activities and that also flexible/non-rigid concepts and materials should be considered in early design. While this case attempted to mimic the flexible nature of a human airway, the actuation and geometrical constraints made this a complex design challenge that was hard to visualize. Trough rough physical modelling and having the opportunity to test often the designers could rapidly generate a functional concept that otherwise could have been hard to come up with.

\subsection{Prototyping with living hinges}

Creating 'flexure patterns' or 'living hinges' is a method utilized to change an object's geometry to enable flexible properties. By cutting or removing material in specific patterns, the mechanical abilities of plate geometries is altered making it possible for rigid materials, such as fibreboard, to bend and flex. Swatches, or premade patterns, can be a time-saver when applying this method. Based on cut orientation and density of the pattern, it is possible to control both axis and radius of deformation. While this method enables rapid generation of flexible concepts using machines such as a laser cutter, design space is constricted to the use of plate geometries. Additionally, both resolution and fidelity are governed by manual assembly of the cut parts.

The project case illustrating this method of prototyping is the conceptual prototyping of a new chest for resuscitation mannequins. The project was challenged with generating new concept ideas for a training mannequin by mimicking the human chest. This required concepts to have similar shape and deformation as a chest would, which inspired the creation of multiple concepts whereas two can be seen in Figure 2. The designers used simple 2D drawings to run the laser cutting operation, which meant little time was spent in virtual prototyping environment. In addition, the speed of fabrication, made it possible for the design team to create multiple design alternatives, hence, adapt quickly to new insights. As seen in Figure 2, the design experimented with different cut patterns that enabled one or two degrees of freedom for the compression mechanism.
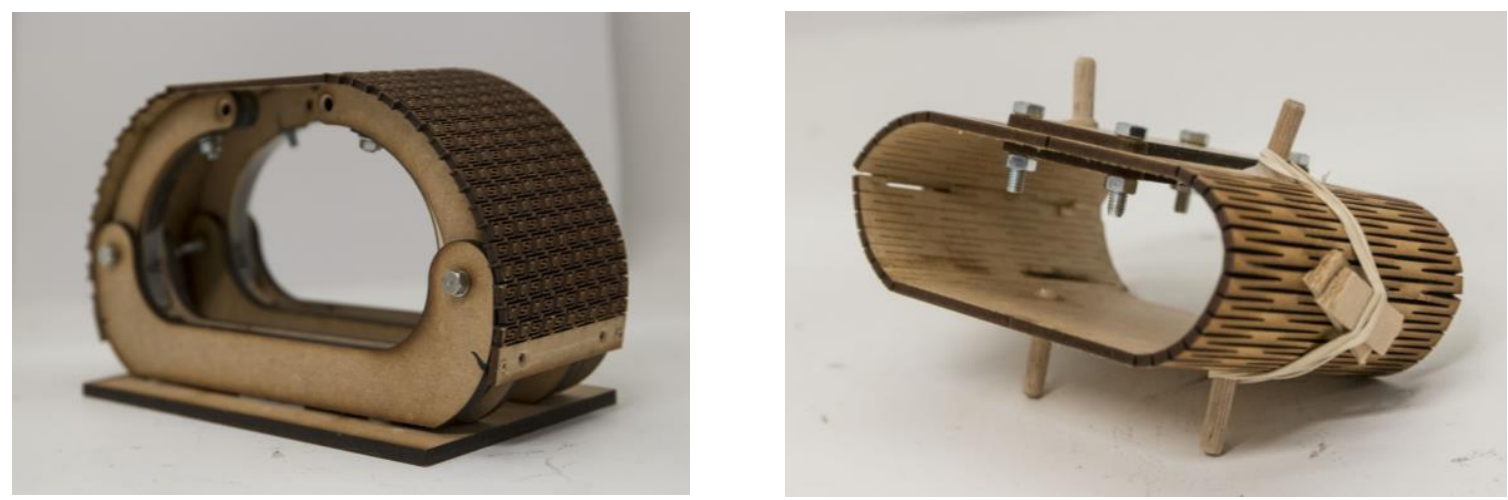

Figure 2. Prototypes built using living hinges 
Using living hinges as a means of creating flexible prototypes is trough the case example shown to be fast and allowing quick design iterations. The method of fabrication benefitted the speed and accuracy of using a laser cutter and having customizable cut patterns enabling this method to create new flexible parts in a matter of minutes. However, both fidelity, resolution and design freedom are for this method governed by the need of a cut pattern to achieve flexible behaviour, rather than leveraging non-rigid material properties. This is further restricting the method to using plate geometries, reducing the design freedom yielded by this method.

\subsection{Prototyping with fused filament fabrication in flexible materials}

Fused Filament Fabrication (FFF), is an additive manufacturing method that uses a layer by layer construction technique of a part modelled in a virtual CAD environment. FFF uses melting of polymer materials on a bed, to build geometrical structures from the bottom up. This method enables the designer to make parts with high fidelity, high resolution and little constraints regarding the geometrical design space, as both layer height and support structures can be optimised to the part geometry. Thermoplastic Polyurethane (TPU) is one example of a flexible FFF material that allows the FFF process to manufacture parts with both complex geometry and flexible properties. As flexible FFF prototypes are melted together, design alterations or rework is highly limited. Therefore, in order to change prototype attributes, changes first must be done virtually before a new part can be manufactured.

The case illustrating this prototyping method, is the development of a spring mechanism for a personal drug mixing container. For this case example, there was a need to reduce mechanical complexity as part count and assembly made the concept prone to jamming and backlash. By drawing on the advantages of FFF with flexible materials it was possible to make a conceptual model, as seen in Figure 3. of high fidelity and resolution, fitting the assembly constraints.

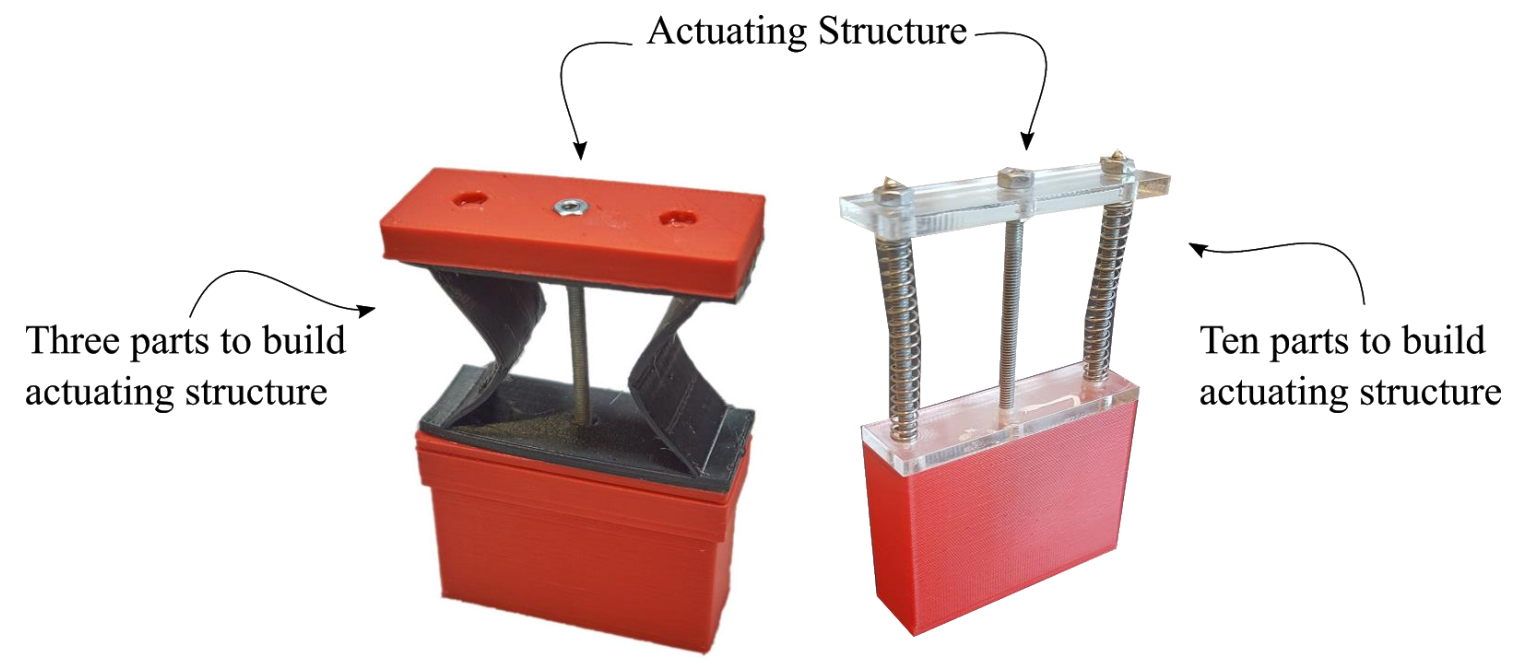

Figure 3. Prototypes built by using fused filament fabrication

In the case it was observed that by utilizing this method of prototyping, designers can simplify a mechanical system that enables easy handling, durability, and robustness. This method of prototyping takes place in both the virtual and physical design space. Therefore, CAD is needed before a physical model is available. An interesting aspect of this project is how the flexible spring structure was tested in the context of the drug container and shown to be unstable because of buckling effects. This could in some cases make the mechanism self-lock and hinder the retraction of the spring. This insight would be hard to obtain without the physical modelling and testing, which highlights the importance of this method as an iterative process. In order to adapt to this insight, the designers had to generate new models. Models that first had to be changed or generated in virtual prototyping environment before creating a new physical model. The virtual rework was done in minutes, but the manufacturing needed hours. The speed of this method is therefore rather low because it does not allow for rapid adjustments and testing. However, the fidelity and resolution it can produce is high. 


\subsection{Prototyping using moulding with flexible materials}

Using moulding as a means of prototyping flexible materials is a high fidelity and resolution way of prototyping parts for testing. The workflow consists of creating a virtual prototype of the intended part or the mould for the part. Generally, using moulding as a method for prototyping is tedious as both moulds and corresponding parts needs to be fabricated. The outcome is however, of high fidelity, and the design freedom the method yields is acceptable as it allows complex outer geometry and surface finish but is restricted concerning internal structures and geometries.

The case example used to demonstrate this prototyping method, a challenge of fabricating and testing a phantom heart in a composite silicone material is considered. This phantom heart was to be used for research on heart flow and ultrasound imaging. To create this phantom, a contrast material was added the silicone to make the part visible on the ultrasonic imaging equipment. For the phantom to achieve flow conditions as found in real heart, the physical model had to replicate key anatomical features of a human heart. High resolution and fidelity were hereby needed to fulfil flow conditions, shape resemblance, and deformation resemblance to a real heart. An additional point to make is that the phantom needed to withstand the internal pressure present as the closed liquid system where to be displayed in air rather than dispersed in liquid. In this case, the heart was first prototyped virtually to create the mould negatives using fused filament fabrication, as shown in Figure 4, and was then moulded using silicone, as shown in Figure 5.

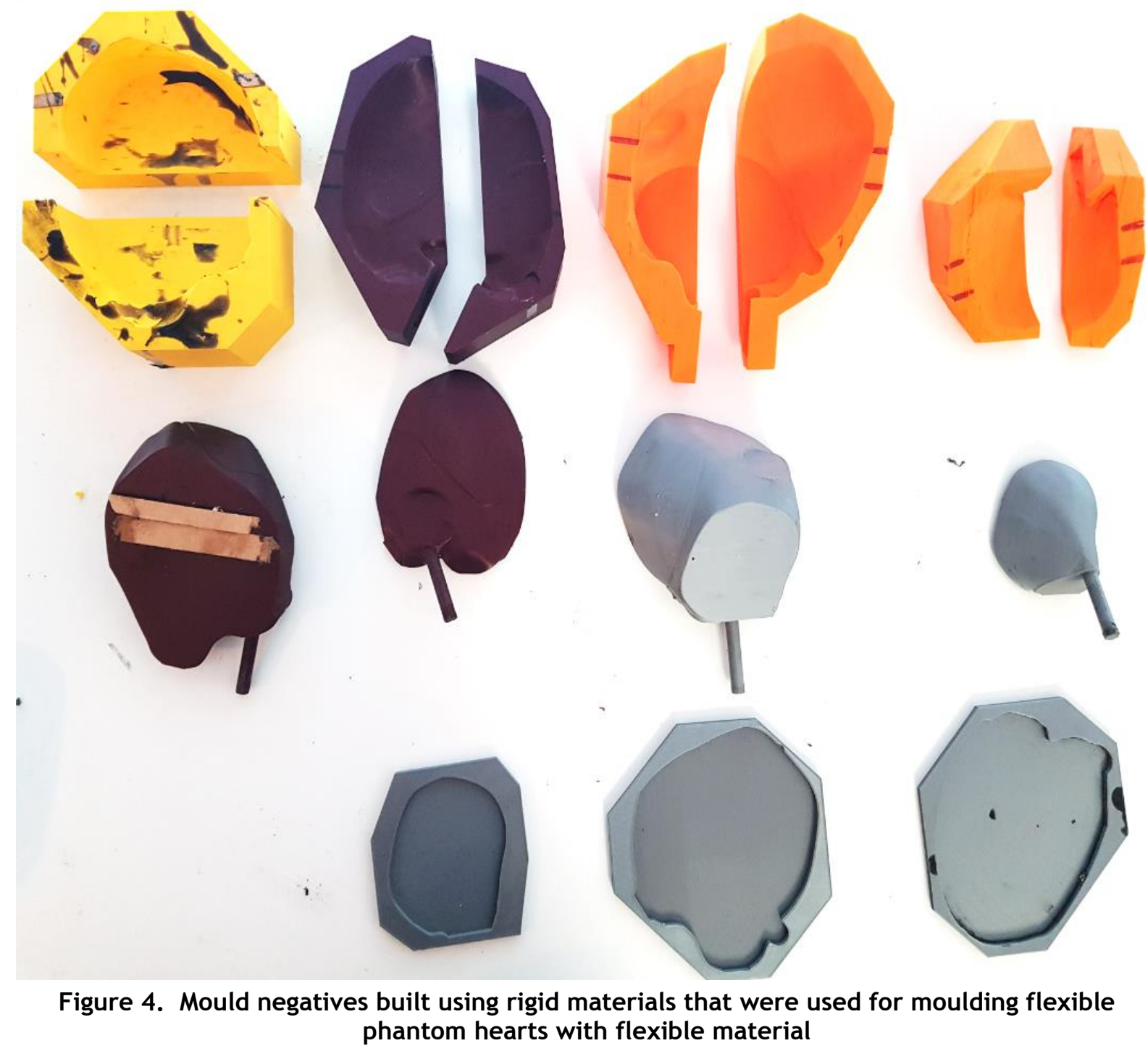



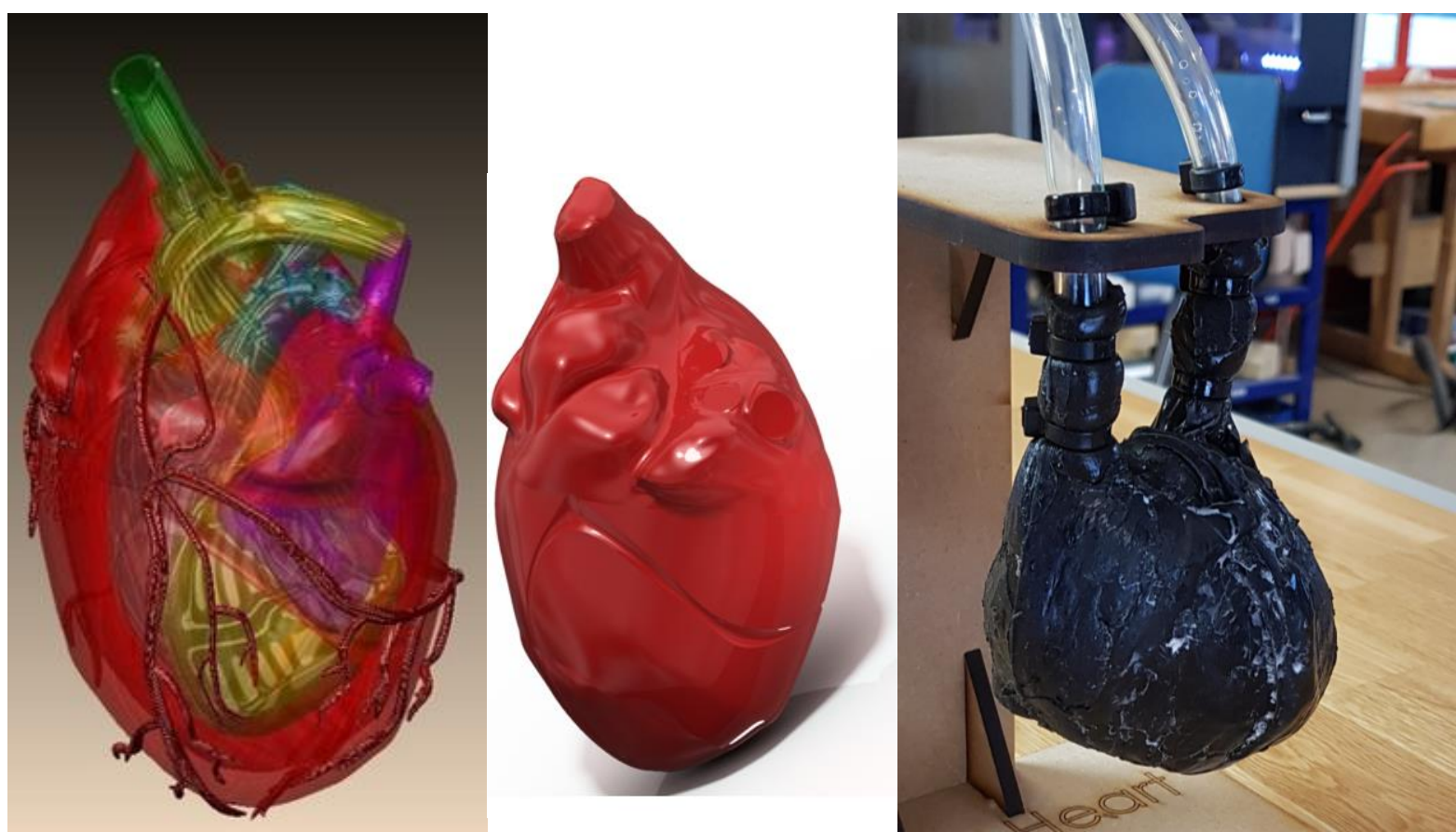

Figure 5. A human heart represented by a virtual prototype (left and centre) and a physical phantom (right) built using moulding with flexible materials

This method of prototyping is relatively slow, as it requires several sequential steps to produce a testable prototype. The production of the mould negatives with FFF (both in CAD and the fabrication itself) is time consuming, and when coupled with the silicone curing time, the per-prototype iteration time until physical testing can start is long. While this case is illustrating mould fabrication using FFF, other methods such as clay casting, manual sculpting and subtractive manufacturing might be more suitable in other cases. As mould fabrication is a means of prototyping moulds, it is in the context of this paper worth noting that efforts in prototyping the moulds affect both attributes of the flexible porotypes as well as activity performance in terms of time and ability to alter designs. One example is how the resolution and fidelity of moulded parts are governed by the surface finish and geometrical design freedom of the moulds, giving FFF advantages of being little constrained in terms of geometry, but subjected to rough surfaces as a result of fabrication technique.

The design freedom of prototyping with moulding is moderate as it can enable any outer geometry but is clearly limited to internal structures and holes making demoulding a challenge. Further it is worth noting that silicone, as one alternative material, only adheres to silicones making it hard to do later rework, or interface using non-silicone materials. Modifications to the prototype itself after the casting is done is therefore both challenging and time consuming. This results in a slow and rigid method considering adapting to insights, as design alterations often would require new mould designs, mould fabrication/alteration, curing time, and additional required assembly.

\section{Discussion}

The presented cases have been investigated, insights have been gathered and evaluated as subjective measurements on the method's effect on design activity and output. This evaluation is presented in Table 1. and subsequently visualised in Figure 6. While the evaluation has elements from established prototype descriptions in literature, it is not intended to be prescriptive or generalisable to other prototyping evaluations, but rather a tool to guide the discussion in this article. This evaluation has been conducted by four coders (from the same research team) that have rated the four project cases, and agreed on the values ranging from 1 to 3 presented in Table 1 . As this rating is only used for discussing and visualizing the findings from the four cases, inter-coder reliability has not been measured. In this section, the authors describe how both prototyping activities and outcome are impacted by the methods employed in the various cases. 
Table 1. The four prototyping methods evaluated in terms of speed, modifications, design freedom, fidelity and resolution. Each method is rated from 1, being low, to 3, being high

\begin{tabular}{|l|l|l|l|l|l|}
\hline & Speed & Modifications & $\begin{array}{l}\text { Design } \\
\text { Freedom }\end{array}$ & Fidelity & Resolution \\
\hline Soft Materials & 3 & 3 & 2 & 1 & 1 \\
\hline Living Hinges & 3 & 2 & 1 & 2 & 2 \\
\hline $\begin{array}{l}\text { Flexible FFF } \\
\text { Materials }\end{array}$ & 2 & 1 & 3 & 3 & 3 \\
\hline $\begin{array}{l}\text { Flexible } \\
\text { Moulding }\end{array}$ & 1 & 1 & 2 & 3 & 3 \\
\hline
\end{tabular}

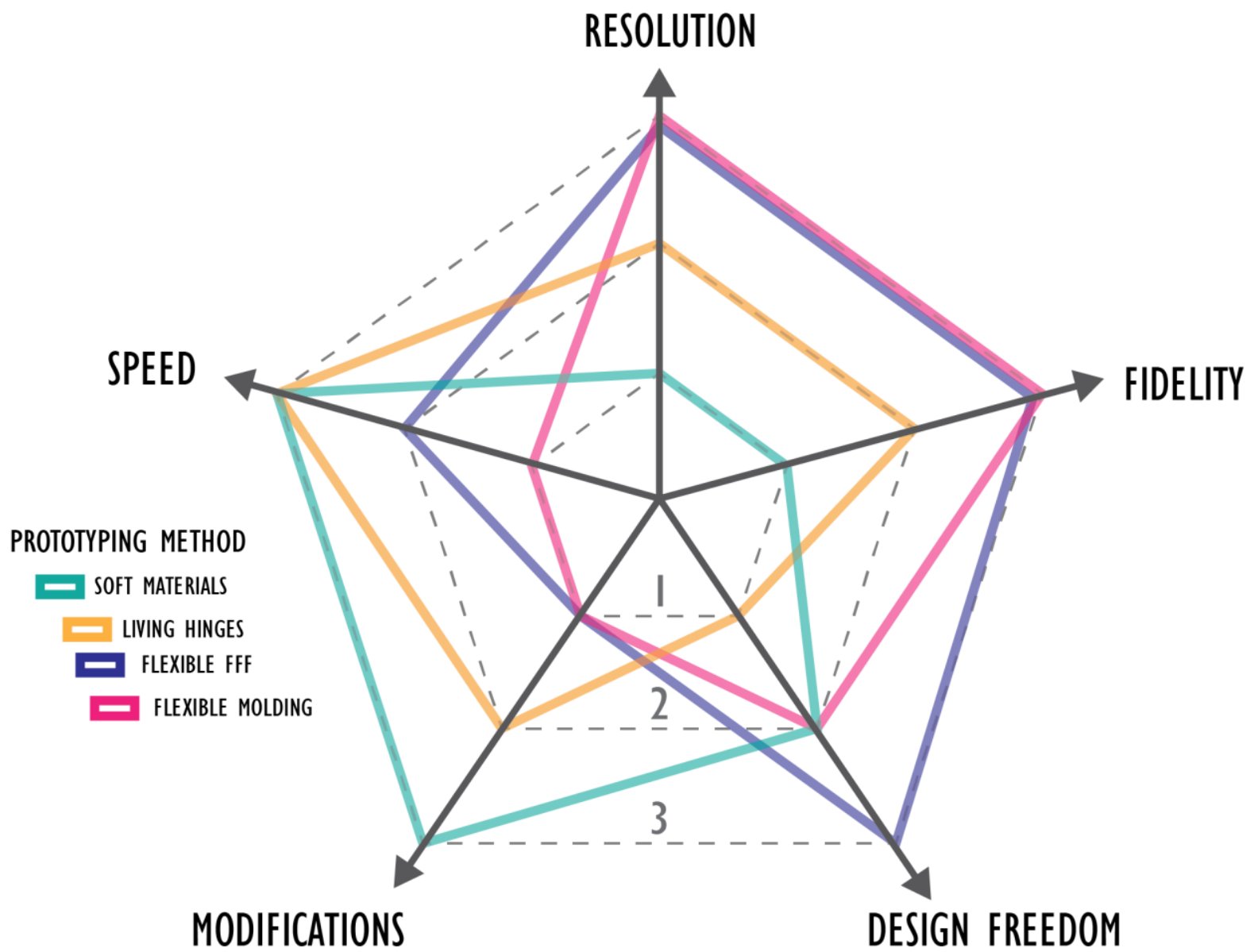

Figure 6. Visualisation of the four prototyping methods evaluated in terms of speed, modifications, design freedom, fidelity and resolution

From inspecting Figure 6, each of the four prototyping methods have distinct differences. Moulding and FFF with flexible materials are both considered methods enabling high fidelity and resolution outcome in terms of accurate and refined prototypes. Because of time and constraints regarding design of moulds and moulding processes, FFF is considered faster and allowing more design freedom than moulding. These methods are however slow and allowing less modifications than both soft prototyping and prototyping using living hinges. The speed of using soft prototyping and living hinges as prototyping approach is high, as these methods require minimal work in CAD environments and not relying on slow machining operations. This makes soft prototyping and using living hinges important methods in gaining insights fast, as learning in this context is a result of creating and testing of prototypes. Further adapting to new insights, soft prototyping is better suited when experimenting and altering designs, while concepts using living hinges require more effort to alter. 
In the early stages of design, prototyping with soft materials and living hinges enable the exploration of new solution spaces. They enable rapid creation of physical prototypes that can be used to test and evaluate non-rigid functions for conceptual designs. The transition to prototyping methods such as FFF and moulding with flexible materials should be done when the required resolution and fidelity is higher. However, these methods require more time and resources for each testable iteration, and should if possible, be delayed to later stages in design. However, when prototyping, the added complexity from using non-rigid materials and creating non-rigid concepts must be acknowledged, possibly creating integration problems at the later stages of design. In addition to opening a potential solution space, flexible mechanical properties make non-rigid concepts harder to predict and replicate. This also applies to the context in which such concepts are intended and how they interface with both users and functional objects. This strengthens the argument for prototyping both early and often to effectively identify and address the uncertainty associated with non-rigid materials, concepts, and context of use in engineering design projects.

Selection of cases and subsequently methods of prototyping with non-rigid materials have in this paper been focused towards exemplifying individual approaches and evaluating them to highlight advantages and limitations for each method. While these methods are not intended as a representation of all methods utilizing non-rigid materials, the selection are highlighting core differences and how they affect prototyping activities and outcome. Since the presented methods are gathered from individual cases, the comparison of methods is limited in terms of workload put into each project, and success criterion and intention for each prototype. However, the cases examples show the applicability of using non-rigid materials and corresponding prototype approaches for leveraging such materials in contexts ranging from low-resolution experiments, to refined models intended for intricate test scenarios.

This paper has evaluated methods of prototyping in order to highlight some of the trade-offs designers must consider when choosing approach for exploring non-rigid concept ideas. With these considerations, selecting appropriate method for the concept to explore, can ensure faster iterations, and being more adaptable to revealed insights. Then designers can decide to invest more time and resources in refined prototypes, when uncertain elements have been revealed trough testing and iterating using more rapid prototyping techniques. This is especially important in the context of non-rigid concepts and in order to leverage non-rigid materials, as predicting mechanical behavior, test conditions, and time and complexity of fabrication all introduce uncertain elements in early design stages.

\section{Conclusion}

In this paper, four methods of prototyping with non-rigid materials have been presented and illustrated trough cases gathered from individual engineering design projects. From these four cases, insights on the utility and implications of using complex non-rigid materials in engineering design practise has been discussed with respect to five specific dimensions; speed, modifications, design freedom, fidelity and resolution. Lastly, implications and normative recommendations for applying these prototyping methods with non-rigid materials in engineering design projects have been discussed.

\section{Acknowledgement}

This research is supported by the Research Council of Norway through its industrial Ph.D. funding scheme, project number 290404.

\section{References}

Auflem, M., Erichsen, J.F. and Steinert, M. (2019), "Exemplifying Prototype-Driven Development through Concepts for Medical Training Simulators", Procedia CIRP, Vol. 84, pp. 572-578. https://doi.org/ 10.1016/j.procir.2019.04.202

Bryan-Kinns, N. and Hamilton, F. (2002), "One for All and All for One?: Case Studies of Using Prototypes in Commercial Projects", Proceedings of the Second Nordic Conference on Human-Computer Interaction, ACM, New York, NY, USA, pp. 91-100.

Buchenau, M. and Suri, J.F. (2000), Experience Prototyping, ACM, New York, NY, USA, pp. 424-433.

Camburn, B.A. et al. (2013), "Methods for Prototyping Strategies in Conceptual Phases of Design: Framework and Experimental Assessment", Volume 5: 25th International Conference on Design Theory and 
Methodology; ASME 2013 Power Transmission and Gearing Conference, presented at the ASME 2013 International Design Engineering Technical Conferences and Computers and Information in Engineering Conference, ASME, Portland, Oregon, USA, p. V005T06A033.

Dow, S.P. et al. (2011), "Parallel prototyping leads to better design results, more divergence, and increased selfefficacy", ACM Transactions on Computer-Human Interaction (TOCHI), Vol. 17 No. 4, pp. 18:1-18:24.

Dow, S.P., Heddleston, K. and Klemmer, S.R. (2009), "The efficacy of prototyping under time constraints", Proceedings of the Seventh ACM Conference on Creativity and Cognition, ACM, pp. 165-174.

Foehr, A.G.C., Stuecheli, M. and Meboldt, M. (2015), "Efficient design evaluation through the combination of numerical and physical computations", DS 80-6 Proceedings of the 20th International Conference on Engineering Design (ICED 15) Vol 6: Design Methods and Tools - Part 2, Milan, Italy (accessed 27-30.07.15).

Houde, S. and Hill, C. (1997), "What do Prototypes Prototype?", In: Handbook of Human-Computer Interaction, Elsevier, pp. 367-381.

Jensen, L.S., Özkil, A.G. and Mortensen, N.H. (2016), "Prototypes in engineering design: Definitions and strategies", 14th International Design Conference International Design Conference, Design Society, pp. 821-830.

Jensen, M.B., Balters, S. and Steinert, M. (2015), "Measuring Prototypes - A Standarized Quantitative Description of Prototypes and their Outcome for Data Collection and Analysis", Proceedings of the 20th International Conference on Engineering Design (ICED15), presented at the International Conference on Engineering Design, Milan.

Kriesi, C. et al. (2016), "Creating Dynamic Requirements through Iteratively Prototyping Critical Functionalities", Procedia CIRP, Vol. 50 No. Supplement C, pp. 790-795.

Lauff, C., Kotys-Schwartz, D. and Rentschler, M.E. (2018), "What is a Prototype? What are the Roles of Prototypes in Companies?" Journal of Mechanical Design, Vol. 140. https://doi.org/10.1115/1.4039340

Leifer, L.J. and Steinert, M. (2011), "Dancing with ambiguity: Causality behavior, design thinking, and tripleloop-learning”, Information Knowledge Systems Management, Vol. 10 No. 1-4, pp. 151-173.

Lim, Y.-K., Stolterman, E. and Tenenberg, J. (2008), "The Anatomy of Prototypes: Prototypes As Filters, Prototypes As Manifestations of Design Ideas", ACM Trans. Comput.-Hum. Interact., Vol. 15 No. 2, pp. 7:1-7:27.

Menold, J.D. (2017). "Prototype for X (PFX): A Prototyping Framework to Support Product Design", Available at: https://etda.libraries.psu.edu/catalog/13760jdm5407 (accessed 7 May 2019).

Schrage, M. (1993), "The Culture(s) of prototyping", Design Management Journal (Former Series), Vol. 4 No. 1, pp. 55-65.

Viswanathan, V. and Linsey, J. (2011). "Design Fixation in Physical Modeling: An Investigation on the Role of Sunk Cost”, pp. 119-130. 